\title{
CHAOTIC MIXING IN MICROFLUIDIC SYSTEMS
}

\author{
Mark A. Stremler, Michael G. Olsen, and Byung H. Jo \\ The Beckman Institute for Advanced Science and Technology \\ University of Illinois at Urbana-Champaign \\ Urbana, IL 61801
}

\author{
Ronald J. Adrian and Hassan Aref \\ Department of Theoretical and Applied Mechanics \\ University of Illinois at Urbana-Champaign \\ Urbana, IL 61801-2983
}

\author{
David J. Beebe \\ Department of Biomedical Engineering \\ University of Wisconsin at Madison \\ Madison, WI 53706-1608
}

\begin{abstract}
Obtaining rapid mixing in microfluidic systems is a problem that must continue to be addressed if microelectromechanical systems are to attain their full potential in commercial markets. We present the paradigm of 'designing for chaos' as a general framework for enhancing mixing in microfluidic applications. This approach is based on a fundamental understanding of the kinematics underlying the mixing process, freeing the MEMS researcher to work with design guidelines instead of empirically determined physical configurations. We have applied this strategy in designing a passive in-line micromixer. Computational and experimental analyses demonstrate the effectiveness of the resulting design in generating chaos in the flow and hence enhancing mixing.
\end{abstract}

\section{INTRODUCTION}

The difficulty in mixing fluids on the microscale lies in the small size of the devices. Despite the small length scales involved, mixing solely by molecular diffusion is too slow for many applications, as the relevant time scales can also be extremely small. Flows at this scale are predominantly laminar, so the efficient mixing obtained in turbulent flows is not practically attainable. Furthermore, fabrication limitations often preclude the use of standard macroscale mixing techniques such as mechanical stirring.

Fortunately, mixing in such flows can be enhanced through 'chaotic advection' [1-3], in which passive fluid particles advected by a periodic, laminar velocity field exhibit chaotic trajectories. Relative to integrable (non-chaotic) advection, chaotic advection enhances stretching and folding of material interfaces. This deformation of fluid-fluid boundaries increases the interfacial area across which diffusion occurs, which increases the mean values of the gradients driving diffusion and leads to more rapid mixing.

A number of tools are available for detecting chaotic advection and measuring its extent, making it possible for a microscale mixer to be 'designed for chaos'. First, a general configuration is chosen based on design guidelines suggested by the theory of chaotic advection. For example, the active mixer of Evans, Liepmann and Pisano [4] is derived from the pulsed source-sink system investigated by Jones and Aref [5]. A mixer is then designed from the general configuration by taking into account the specific application and the fabrication constraints. The resulting device is modeled numerically, and diagnostics are computed to verify the occurrence of chaotic advection. Iterations can then be made on the design to optimize the amount of chaos present. Finally, the device is tested experimentally to verify that mixing is enhanced. The use of this paradigm for designing a passive mixer is illustrated in the following sections.

\section{DEVICE DESIGN}

Passive mixing in a single microchannel is attractive for a number of reasons. First of all, a passive mixing scheme, which relies solely on a constant flow source, is generally more robust and easier to implement than an active mixing scheme, which relies on exerting some form of control over the flow field through such means as moving parts or varying pressure gradients. Secondly, a single channel maintains a relatively constant cross-section, which results in lower strain rates than if the flow is split into multiple smaller streams or forced through small orifices. Thus the existing work on chaotic advection is investigated to determine the guidelines for designing such a mixer.

It has been shown with a simple analytical model that flow in a 'twisted pipe' can contain significant amounts of chaotic advection [6]. The basic configuration consists of a sequence of pipe bends, with successive bends oriented along different planes. This result has been applied successfully to heat transfer enhancement on the macroscale [7-9], and the goal is to now implement this design on the microscale.

Previous studies of the 'twisted pipe' have only considered flow in circular pipes arranged in curved three-dimensional configurations. Fabrication limitations lead us to consider instead the related orthogonal geometry shown in figure 1, which can be constructed using a variety of techniques. The channels used in this study are fabricated of polydimethylsiloxane (PDMS) using a micro-molding technique [10]; a variation on the design in figure 1 has also been fabricated and tested in silicon [11].

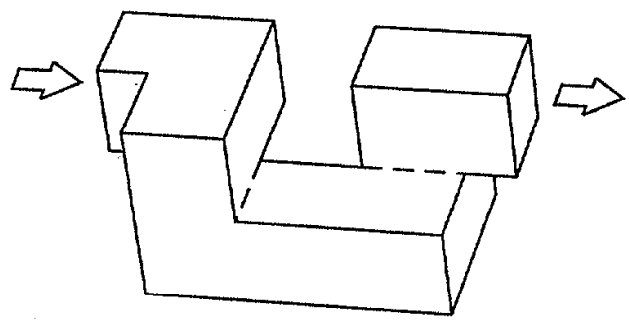

Figure 1. One segment of the ' $3 D$ serpentine' mixer. The fabricated mixer consists of 11 segments placed end-to-end, and the channel cross-section is $300 \mu \mathrm{m} \times 300 \mu \mathrm{m}$. 
An alternative configuration is the 'zig-zag' channel investigated by Branebjerg, Fabius, and Gravesen [12]. However, theoretical results for the 'twisted pipe' suggest that this channel design will generate little or no chaotic advection. We investigate the similar 'square-wave' geometry shown in figure 2 and compare its mixing capabilities with the $3 D$ serpentine channel to illustrate the effectiveness of producing chaos in the flow.

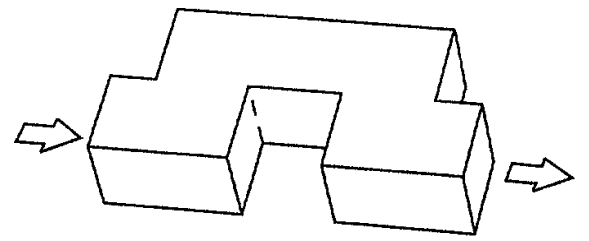

Figure 2. One segment of the 'square-wave' mixer. The fabricated mixer consists of 14 segments placed end-to-end, and the channel cross-section is again $300 \mu m \times 300 \mu m$.

\section{NUMERICAL MODELING}

While theory can suggest or predict the occurrence of chaotic advection, the amount of chaos present can only be determined for specific examples. A standard tool for detecting chaos in spatially or temporally periodic systems is the Poincaré section [3]. A schematic of how this section is generated for spatially periodic systems is shown in figure 3 . The Poincaré section consists of patterns of points formed by passive particles advected through the periodic flow field. Chaotic advection is present in those portions of the section where the resulting pattern is random.

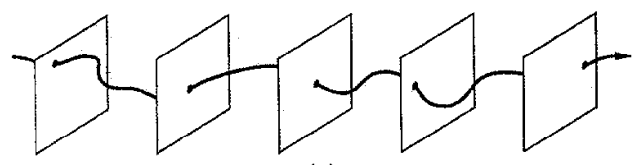

(a)

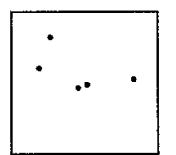

(b)
Figure 3. Generation of a Poincaré section in a spatially periodic system. (a) A passive particle is advected by the periodic velocity field and passes through a series of periodic planes in the system. For the mixers considered here the planes are located at the exit (or entrance) of each mixing segment. The position at which the particle passes through each plane is recorded. (b) All of the positions are transferred to a single plane, which is the Poincaré section. Regular patterns in the Poincaré section indicate integrable (non-chaotic) behavior, while random patterns indicate chaotic behavior.

In order to generate Poincaré sections in these channels, the velocity fields are first computed on a grid using CFD-ACE, a commercial finite-volume code from CFD Research Corporation. The channels are modeled as infinitely long by enforcing periodic boundary conditions at the inlet and exit of a single mixer segment. An important parameter in these flows is the Reynolus number, $\operatorname{Re}=\mathrm{UL} / \mathrm{v}$, which gives the relative importance of inertia and viscosity in the system in terms of the mean channel vclocity $U$, the kinematic viscosity $v$ of the fluid, and the width $L$ of the channel cross-section. For waterbased flows in these channels, $\operatorname{Re}=10$ corresponds to a flow rate of approximately $0.2 \mathrm{~mL} / \mathrm{min}$. Velocity fields are computed at various Reynolds numbers by varying the specified pressure drop across one segment of the mixer. Particle traces and the corresponding Poincare section are then generated by integrating numerically through the discrete velocity field using an adaptive Runga-Kutta scheme. The velocity at any point within the channel is determined by polynomial interpolation.

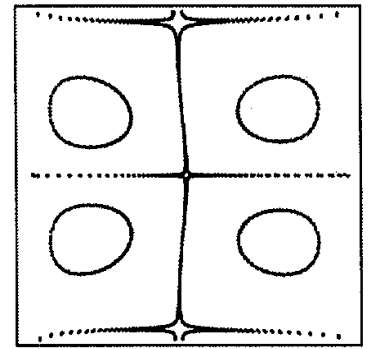

(a)

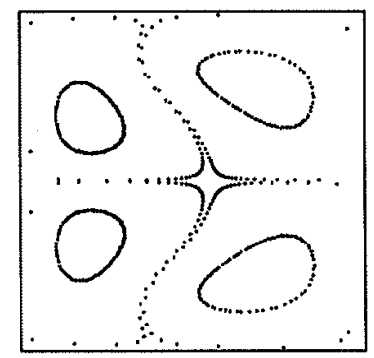

(c)

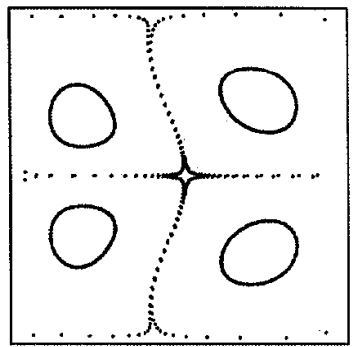

(b)

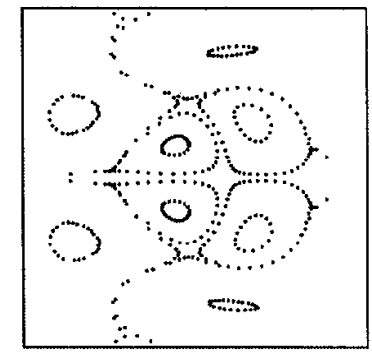

(d)
Figure 4. Poincare maps in the square-wave mixer at (a) $R e=10$, (b) $R e=20$, (c) $R e=30$, and (d) $R e=50$. Each closed curve shown is generated by tracing a single particle. Those curves passing very close to the boundary are not shown as closed due to numerical error that occurs in following particles near the wall of the channel. While the complexity of the advection does increase with increasing Reynolds number, no chaos is present.

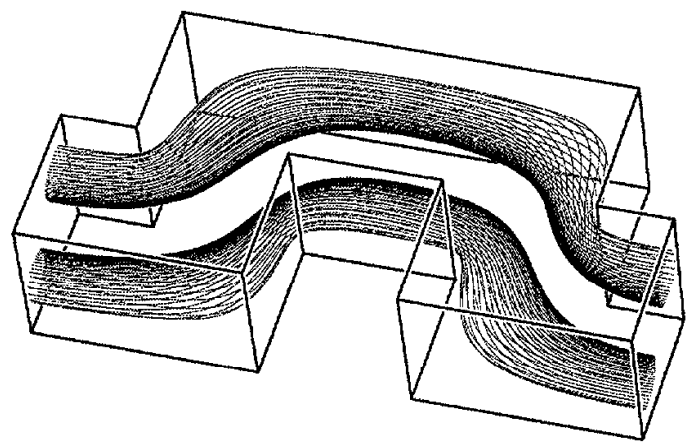

Figure 5. Particle traces in the square-wave mixer at a Reynolds number of 10 . The lower tube corresponds to initial conditions lying on the lower left 'circle' in figure $4(a)$, and the upper tube to the upper right 'circle' in figure $4(a)$.

Poincare sections in the square-wave channel for Reynolds numbers from 10 to 50 are shown in figure 4 . In each case no chaotic advection appears, as is expected from the analytical results [6]. The reason a non-chaotic flow is expected to mix poorly is illustrated in figure 5 . Closed curves in the Poincaré section correspond to 'stream tubes' in the flow that are barriers 
across which fluid particles cannot move. Thus a mechanism such as diffusion is required to mix between the two tubes shown in figure 5.

Poincare sections in the 3D serpentine channel for Reynolds numbers from 1 to 20 are shown in figure 6 . In contrast to the square-wave mixer, chaotic advection does occur in the flow for the Reynolds numbers considered, with significant amounts of chaos present already at $R e=10$. Thus it is expected that the $3 \mathrm{D}$ serpentine mixer will perform significantly better than the square-wave mixer for moderate Reynolds numbers (roughly for $\mathrm{Re} \geq 10$ ).

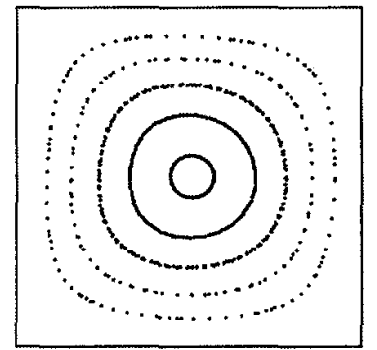

(a)

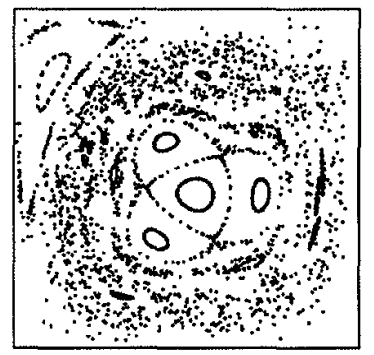

(c)

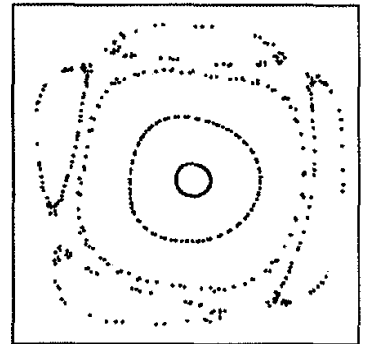

(b)

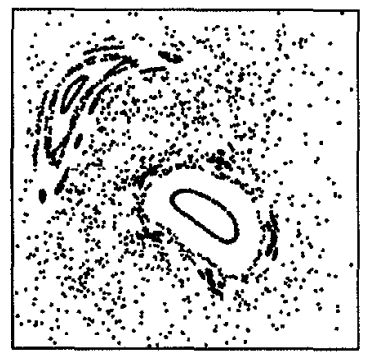

(d)
Figure 6. Poincaré maps in the $3 D$ serpentine mixer at (a) $R e=1$, (b) $R e=5$, (c) $R e=10$, and (d) $R e=20$. At low Reynolds numbers the Poincare sections are dominated by regular closed curves, and the advection is predominantly non-chaotic. However, for $R e \geq 10$ the Poincaré sections contain large regions of chaos. In (c) and $(d)$ the particle patterns forming the chaotic regions are generated by following only 1 or 2 particles.

\section{EXPERIMENTAL COMPARISONS}

Mixing in the channels is evaluated experimentally by observing the color change of a $\mathrm{pH}$ indicator as it flows through the channel. Two separate fluid streams flow into each channel through a T-junction. One stream contains phenolphthalein dissolved in a mix of 50\% ethyl alcohol and 50\% de-ionized water with a concentration of $0.016 \mathrm{M}$. The second stream contains $98.3 \%$ sodium hydroxide pellets also dissolved in a $50 / 50$ mix of alcohol and water, giving a $\mathrm{pH}$ of approximately 13.

The phenolphthalein and sodium hydroxide streams are introduced into the mixer from two water columns. After the two colorless streams come in contact, both sodium hydroxide and phenolphthalein begin to diffuse. The reaction time of the phenolphthalein is negligible, although the ratio of reacted to unreacted phenolphthalein at any point within the device depends on the local $\mathrm{pH}$ value in a non-trivial way [13]. Thus, the amount of reacted phenolphthalein produced depends on the diffusivities and initial concentrations of both phenolphthalein and sodium hydroxide.

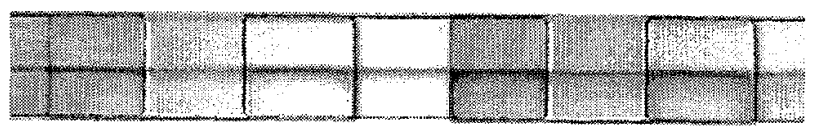

(a)

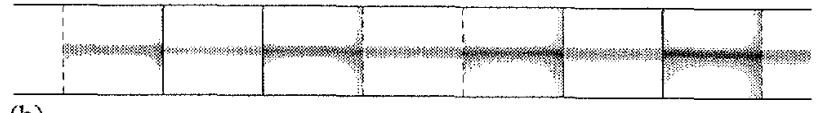

(b)

Figure 7. (a) Experimental results and (b) computational results for diffusion in the first two segments of the square-wave mixer at a Reynolds number of approximately 10. Flow is from left to right. The view is from the front of the mixer as oriented in figure 2. At the mixer inlet the top half of the channel contains the $\mathrm{NaOH}$ stream and the bottom half the phenolphthalein stream. The reacted phenolphthalein appears as a dark product.

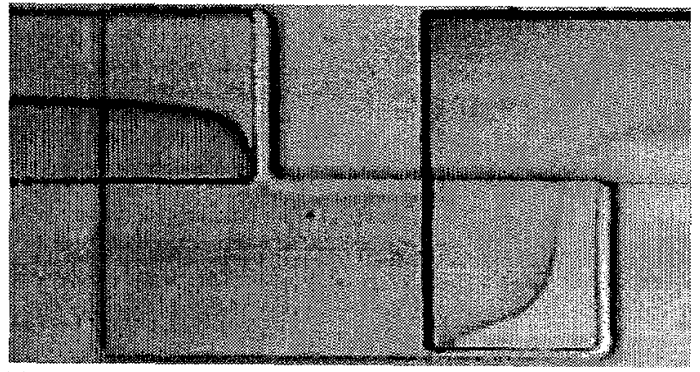

(a)

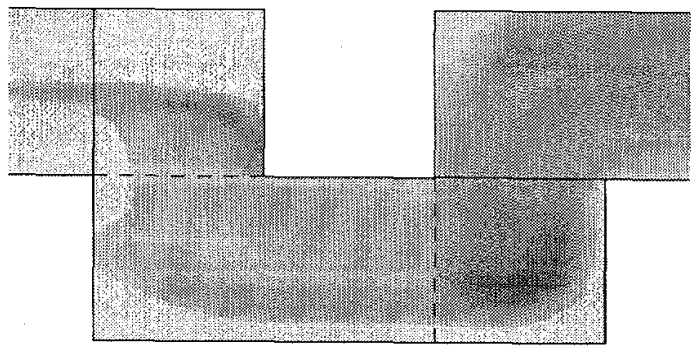

(b)

Figure 8. (a) Experimental results and (b) computational results for diffusion in the first segment of the $3 D$ serpentine mixer at a Reynolds number of approximately. 10. Flow is from left to right. The view is from the front of the mixer as oriented in figure 1 .

The mixing ability of the device is determined qualitatively by observing the color change of the phenolphthalein during the mixing process. Images of the reacted phenolphthalein are captured through an Olympus BX60 microscope at $4 \mathrm{X}$ magnification with a Sony 8-bil CCD camera. The microscope objective used has a depth-of-focus of $175 \mu \mathrm{m}$. Although by this definition the entire channel depth is not in perfect focus, blurring of features in the flow is negligible. Illumination comes from a halogen light source mounted behind the mixer. Since the halogen lamp produces incoherent light, the light waves scattered by two molecules are assumed to have additive intensities when the images of the molecules overlap. Thus the 
intensity of red observed in the channel is proportional to the amount of reacted phenolphthalein present.

As shown in figures 7 and 8 , there is good qualitative agreement between the experimental results and a simple computational diffusion model, which demonstrates the validity of the numerical model and lends credibility to the numerical diagnosis of chaos presented in figures 4 and 6 .

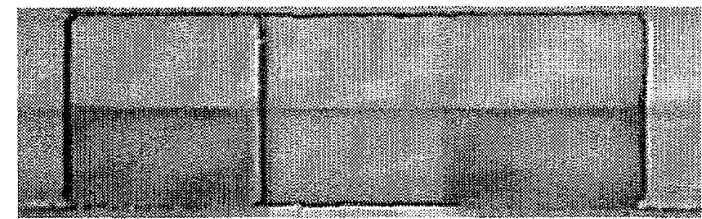

(a)

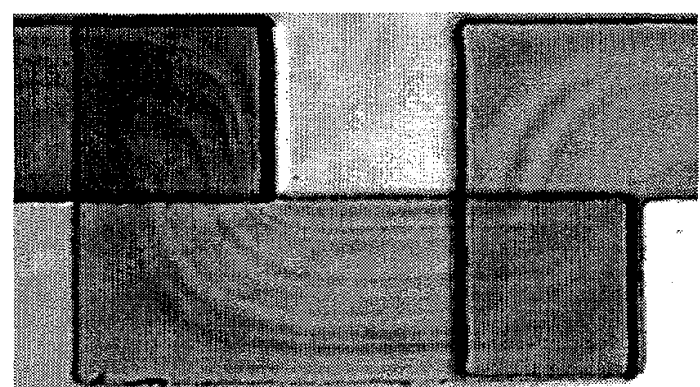

(b)

Figure 9. Comparison of the mixing results at the last mixing segment in (a) the square-wave mixer and (b) the $3 D$ serpentine mixer. The fluid has traveled approximately the same distance in each mixer at the segments shown. Stretching and folding of the material interface is apparent in $(b)$.

The mixing capabilities of these two channels are compared experimentally in figure 9. In the square-wave mixer product is observed only in the bottom half of the channel, and much of the product is concentrated at the centerline. In contrast, the 3D serpentine mixer produces more product, and this product is distributed throughout the channel cross-section. Thus, designing for chaos has resulted in a mixer that performs better than one that does not produce chaotic advection.

\section{CONCLUSIONS}

The paradigm of dcsigning for chaos in microfluidic systems gives the MEMS researcher the freedom to work within a flexible design framework instead of relying solely on the use of empirically determined devices. The validity of this approach has been demonstrated in the design of a passive, inline micromixer. The resulting device enhances mixing significantly relative to a similar in-line mixer that does not produce chaotic advection in the flow. This approach of 'designing for chaos' is not limited to passive in-line devices. Indeed, there are a variety of mixers that can be developed using this framework.

\section{ACKNOWLEDGEMENTS}

This work was supported under a grant from DARPA-MTO (\# F33615-98-1-2853, Program manager: Dr. Abraham Lee).

\section{REFERENCES}

1. H. Aref, "Stirring by chaotic advection", J. Fluid Mech., 143, 1-21 (1984).

2. H. Aref, "Chaotic advection of fluid particles", Phil. Trans. R. Soc. Lond. A, 333, 273-288 (1990).

3. J. M. Ottino, The Kinematics of Mixing: Stretching, Chass, and Transport, Cambridge University Press (1989).

4. J. Evans, D. Liepmann, and A. P. Pisano, "Planar laminar mixer", Proc. IEEE Workshop Micro Electro Mech. Sys. (MEMS '97), Nagoya, Japan (1997), pp. 96-101.

5. S. W. Jones and H. Aref, "Chaotic advection in pulsed source-sink systems", Phys. Fluids, 31, 469-485 (1988).

6. S. W. Jones, O. M. Thomas, and H. Aref, "Chaotic advection by laminar flow in a twisted pipe", J. Fluid Mech., 209, 335 357 (1989).

7. N. Acharya, M. Sen, and H.-C. Chang, "Heat transfer enhancement in coiled tubes by chaotic mixing", Int. J. Heat Mass Trans., 35, 2475-2489 (1992).

8. D. Sawyers, M. Sen, and H.-C. Chang, "Effect of chaotic interfacial stretching on bimolecular chemical reaction in helical-coil reactors", Chem. Eng. J., 64, 129-139 (1996).

9. A. Mokrani, C. Castelain, and H. Peerhossaini, "The effect of chaotic advection on heat transfer", Int. J. Heat Mass Trans., 40, 3089-3104 (1997).

10. B. H. Jo, L. VanLergerghe, K. Motsegood, and D. J. Beebe, "Fabrication of three-dimensional microfluidic systems by stacking molded PDMS layers", Proc. SPIE Symp. Micromachining Microfabrication, Santa Clara, CA (1999), pp. 222-229.

11. R. H. Liu, M. A. Stremler, K. V. Sharp, M. G. Olsen, J. G. Santiago, R. J. Adrian, H. Aref and D. J. Beebe, "Passive mixing in a three-dimensional serpentine microchannel", $J$. Microelectomechanical Sys., (to appear).

12. J. Branebjerg, B. Fabius, and P. Gravesen, "Application of miniature analyzers: from microfluidic components to $\mu \mathrm{TAS}$ ", Proc. Micro Total Anal. Sys. Workshop ( $\mu$ TAS '94), Enschede, The Netherlands (1994), pp. 141-151.

13. S. Zhang, S. P. Schneider, and S. H. Collicott, "Quantitative molecular-mixing measurments using digital processing of adsorption images", Exp. in Fluids, 19, 319-327 (1995). 